\title{
Plasma long non-coding RNA MALAT1 is associated with distant metastasis in patients with epithelial ovarian cancer
}

\author{
QINGJUAN CHEN, YONGYONG SU, XIAOPENG HE, WEIAN ZHAO, CAIXIA WU, \\ WEIBO ZHANG, XIAOMIN SI, BINGWEI DONG, LIANYING ZHAO, YUFANG GAO, \\ XIAOWEN YANG, JIANHUI CHEN, JIAN LU, XIMIN QIAO and YUCHEN ZHANG
}

Department of Medical Oncology, Xianyang Central Hospital, Xianyang, Shaanxi 712000, P.R. China

Received February 8, 2015; Accepted May 26, 2016

DOI: $10.3892 / 01.2016 .4800$

\begin{abstract}
Human metastasis-associated lung adenocarcinoma transcript 1 (MALAT1) is a newly identified metastasis-associated long non-coding RNA. In a previous study, it was identified that plasma levels of MALAT1 were significantly increased in gastric cancer patients with metastasis compared with gastric cancer patients without metastasis and healthy control individuals. However, it is unclear whether plasma levels of MALAT1 may act as a biomarker for evaluating the development of metastasis in epithelial ovarian cancer (EOC). In the present study, groups that consisted of 47 patients with EOC and metastasis (EOC/DM), 47 patients with EOC without metastasis (EOC/NDM), and 47 healthy control (HC) individuals were established. Reverse transcription-quantitative polymerase chain reaction (RT-qPCR) was used to detect the level of plasma MALAT1 in these groups. The results showed that levels of plasma MALAT1 were significantly increased in the EOC/DM group compared with the EOC/NDM and HC groups $(\mathrm{P}<0.001)$. Receiver operating characteristic (ROC) analysis indicated that plasma MALAT1 yielded an area under the curve (AUC) of 0.820 [95\% confidence interval (CI), 0.734-0.905; $\mathrm{P}<0.001$ ], distinguishing between EOC/DM and EOC/NDM. ROC analysis also yielded an AUC of 0.884 (95\% CI, 0.820-0.949; $\mathrm{P}<0.001$ ), with $89.4 \%$ sensitivity and $72.3 \%$ specificity for distinguishing between EOC/DM and $\mathrm{HC}$. Furthermore, multivariate analysis indicated that
\end{abstract}

Correspondence to: Mr. Yuchen Zhang, Department of Medical Oncology, Xianyang Central Hospital, 78 Renmin East Road, Xianyang, Shaanxi 712000, P.R. China

E-mail: 312871805@qq.com

Abbreviations: lncRNAs, long non-coding RNAs; MALAT1, metastasis-associated lung adenocarcinoma transcript 1; EOC, epithelial ovarian cancer; EOC/DM, epithelial ovarian cancer with metastasis; EOC/NDM, epithelial ovarian cancer without metastasis; HC, healthy controls; RT-qPCR, reverse transcription-quantitative polymerase chain reaction; CA125, carbohydrate antigen 125

Key words: long non-coding RNA, MALAT1, epithelial ovarian cancer, distant metastasis overexpression of MALAT1, differentiation (poor), tumor-node-metastasis stage (IV), lymph node metastasis (N3), peritoneal invasion (present) and higher serum carbohydrate antigen 125 levels were independent predictors of survival (hazard ratio, 3.322; $\mathrm{P}=0.028$ ) in patients with EOC. Kaplan-Meier analysis revealed that patients with increased MALAT1 expression had a poorer disease-free survival time. In conclusion, the levels of plasma MALAT1 may act as a valuable biomarker for the diagnosis of metastasis.

\section{Introduction}

Although the incidence of ovarian cancer ranked eighth and the mortality ranked seventh in females worldwide in 2008 (1), ovarian cancer remains one of the most prevalent, deadly and costly gynecological cancers (2). Epithelial ovarian cancer (EOC) accounts for almost $90 \%$ of all ovarian tumors (3-5) and is characterized by advanced-stage presentation, multiple organ metastases, peritoneal dissemination and refractory ascites when diagnosed (6). Although progress in early detection technology (transvaginal ultrasound, CA125 examination, computed tomography and BRCA gene mutation detection) and intervention management (surgical management for borderline ovarian tumors, ovariectomy for high-risk women) has decreased the mortality rate in multiple fields, advanced-stage diseases, particularly those that have metastasized, continue to frequently progress to recurrent disease and have a poor prognosis, with an average disease progression-free survival time of 18 months and a 5-year overall survival rate of $<30 \%$ (7-9).

At present, the diagnosis of metastasis depends on detection by ultrasound and computed tomography scanning. As a supplemental diagnostic marker, the level of carbohydrate antigen 125 (CA125) is also considered (10). However, these measures have limited utility due to their inadequate sensitivity and specificity (11); the vast majority of metastases continue to be found during surgery due to false negative tests (12). Therefore, the development of novel biomarkers for early detection of EOC with metastasis (EOC/DM) is imperative and urgent.

There are a large number of non-protein-coding RNAs in the human genome, which do not encode proteins and were once considered as 'noise' in genome transcription $(13,14)$. Previous studies have found that these non-protein coding RNAs regulate cell differentiation, growth and metabolism, 
in addition to numerous other biological processes, at the transcriptional, post-transcriptional or translational levels, and at the DNA, RNA or protein levels (15-18). Metastasis-associated lung adenocarcinoma transcript 1 (MALAT1) is one of the few well-studied long non-coding RNAs (lncRNAs), which presents high expression and enhanced activity in numerous tumor tissues and cells (19-21), playing a critical role in tumor invasion and metastasis (22). Studies have verified that MALAT1 is closely associated with metastasis in non-small cell lung cancer (NSCLC) $(23,24)$ and several other human solid tumors, including colorectal cancer, osteosarcoma, bladder cancer and gallbladder cancer (25-28). At present, there is a lack of research concerning plasma MALAT1 expression in patients with metastatic EOC.

\section{Materials and methods}

Patients and samples. A case-control study was designed to identify whether MALAT1 could be used as a substitute marker for EOC/DM. All patients were enrolled from the Xianyang Central Hospital (Xianyang, Shaanxi, China) between January 2010 and November 2014. The present study was approved by the Clinical Research Ethics Committee of Xianyang Central Hospital and all individuals provided written informed consent. Plasma preparation and RNA isolation were performed on the clinical samples obtained.

The present cohort totaled 141 female participants, consisting of 47 patients with EOC/DM, 47 patients with EOC without metastasis (EOC/NDM) and 47 healthy control (HC) individuals. The clinical stage of EOCs was conducted according to the tumor-node-metastasis (TNM) (29) and Federation of Gynecology and Obstetrics (30) staging systems. The inclusion criteria for EOC/DM were: Liver, lung or other distant organ metastasis identified by imaging and confirmed pathologically. The 47 patients with EOC/NDM had no pathological evidence of distant organ metastasis; HC individuals were also recruited as patients who underwent routine medical examination and had no evidence of tumor disease in the same period. The HC individuals were matched to the patients based on age and gender. The baseline features of the patients and $\mathrm{HC}$ individuals are summarized in Table I.

Plasma preparation. All plasma samples were collected prior to any therapeutic procedure, such as surgery, chemotherapy or radiotherapy. Plasma sample preparation and total RNA isolation were performed as previously described (31). In brief, $5 \mathrm{ml}$ venous blood was collected in an EDTA gel tube, and the separation procedure was performed within $2 \mathrm{~h}$ of sample collection. Plasma samples were centrifuged at $1,000 \mathrm{x} \mathrm{g}$ for $10 \mathrm{~min}$, followed by $13,000 \mathrm{x} \mathrm{g}$ for a further $10 \mathrm{~min}$ at $4^{\circ} \mathrm{C}$. The supernatant plasma was then separated, split into $250 \mu \mathrm{l}$ aliquots, and frozen at $-80^{\circ} \mathrm{C}$ until use.

RNA preparation, reverse transcription $(R T)$ and $R T$-quantitative polymerase chain reaction $(q P C R)$. Total RNA was extracted from plasma using TRIzol LS reagent (Invitrogen; Thermo Fisher Scientific, Inc., Waltham, MA, USA), following the manufacturer's protocol with minor modifications (when precipitating RNA, RNase-free glycogen was not added as a carrier to the aqueous phase). RNA was removed from genomic DNA and reverse transcribed to cDNA using a PrimeScript ${ }^{\circledR}$ RT reagent Kit with gDNA Eraser (catalog no., DRR047; Takara Bio, Inc., Otsu, Shiga, Japan). Subsequently, RT-qPCR was performed using SYBR ${ }^{\circledR}$ Premix Ex Taq II (Perfect Real Time; catalog no., DRR081; Takara Bio, Inc.) on ABI7500 (Applied Biosystems; Thermo Fisher Scientific, Inc.). To examine the expression of MALAT1 in EOC, RT-qPCR amplification was performed using the following specific primers: MALAT1 sense, 5'-CTTCCCTAGGGG ATTTCAGG-3' and antisense, 5'-GCCCACAGGAACAAG TCCTA-3' (32). $\beta$-actin was used as the internal reference, and the primers were as follows: $\beta$-actin sense, 5'-CTTAGT TGCGTTACACCCTTTCTTG-3' and antisense, 5'-CTGTCA CCTTCACCGTTCCAGTTT-3'. Amplification was performed under the following conditions: $95^{\circ} \mathrm{C}$ for $30 \mathrm{sec}$, followed by 40 cycles of $95^{\circ} \mathrm{C}$ for $5 \mathrm{sec}, 60^{\circ} \mathrm{C}$ for $30 \mathrm{sec}$ and $72^{\circ} \mathrm{C}$ for $30 \mathrm{sec}$. Melting curve analysis was performed as follows: $95^{\circ} \mathrm{C}$ for $0 \mathrm{sec}$ (at $20^{\circ} \mathrm{C} / \mathrm{sec}$ from $72-95^{\circ} \mathrm{C}$ ); and $65^{\circ} \mathrm{C}$ for $15 \mathrm{sec}$ to $95^{\circ} \mathrm{C}$ for $0 \sec \left(\right.$ at $0.1^{\circ} \mathrm{C} / \mathrm{sec}$ ). Experiments were performed in triplicate in the same reaction. The results of RT-qPCR experiments were calculated using the $2^{-\Delta \Delta \mathrm{Cq}}$ method (33).

Statistical analysis. Normalized MALAT1 levels were demonstrated as $\Delta \mathrm{Cq}$, with $\Delta \mathrm{Cq}=\mathrm{Cq}$ (MALAT1) $-\mathrm{Cq}$ ( $\beta$-actin). The difference between groups was analyzed by the Mann-Whitney or Kruskal-Wallis tests. Receiver operating characteristic (ROC) curves and the area under the curve (AUC) were conducted to determine the diagnostic power of plasma MALAT1 in distant metastasis. COX analysis was used to assess the clinicopathological factors that were associated with survival in the EOC patients. The clinicopathological features were assessed by one-way analysis of variance, Student's t-test or $\chi^{2}$ test, as appropriate. For the post-hoc test, multiple comparisons between the groups were performed using the Student-Newman-Keuls method. $\mathrm{P}<0.05$ was considered to indicate a statistically significant difference. Statistical analyses were conducted using SPSS 16.0 software (SPSS Inc., Chicago, IL, USA). Graphs and charts were created in GraphPad Prism (version 5.00 for Windows; GraphPad Software, Inc., La Jolla, CA, USA) and MedCalc software (version 12.3.0.0; MedCalc Software, Ostend, Belgium).

\section{Results}

Evaluation of clinical features in study groups. The baseline clinicopathological features of the participants enrolled in the present study are summarized in Table I. All 94 EOC patients and $47 \mathrm{HC}$ individuals did not differ in age or gender. In addition, there was no significant difference in the distribution of TNM stage, pathological differentiation, serum CA125 level, peritoneal invasion status, lymph node metastasis and metastatic sites between the EOC/DM and the EOC/NDM groups.

MALAT1 expression was upregulated in the plasma of metastatic ovarian epithelial carcinoma patients. The expression levels of plasma MALAT1 in 94 EOC patients and $47 \mathrm{HC}$ individuals were examined by RT-qPCR. The findings demonstrated that, compared with the EOC/NDM and HC groups, the expression of plasma MALAT1 was significantly higher 
Table I. Clinicopathological features of EOC patients and HC individuals.

\begin{tabular}{|c|c|c|c|}
\hline Variables & EOC/DM, n (\%) & EOC/NDM, n (\%) & $\mathrm{HC}, \mathrm{n}(\%)$ \\
\hline Total $^{\mathrm{a}}$ & $47(100.0)$ & $47(100.0)$ & $47(100.0)$ \\
\hline \multicolumn{4}{|l|}{ Age, years } \\
\hline Median & 58 & 59 & 59 \\
\hline Range & $49-64$ & $48-66$ & $48-68$ \\
\hline \multicolumn{4}{|l|}{ TNM stage } \\
\hline III & $0(0.0)$ & $4(8.5)$ & \\
\hline IV & $47(100.0)$ & $43(91.5)$ & \\
\hline \multicolumn{4}{|c|}{ Pathology differentiation } \\
\hline Well & $5(10.6)$ & 7 (14.9) & \\
\hline Moderate & $17(36.2)$ & $19(40.4)$ & \\
\hline Poor & $25(53.2)$ & $21(44.7)$ & \\
\hline \multicolumn{4}{|c|}{ Serum CA125 level } \\
\hline$\leq 35 \mathrm{U} / \mathrm{ml}$ & $2(4.3)$ & $3(6.4)$ & \\
\hline$>35 \mathrm{U} / \mathrm{ml}$ & 45 (95.7) & $44(93.6)$ & \\
\hline \multicolumn{4}{|c|}{ Peritoneal invasion } \\
\hline Absence & $27(57.4)$ & $30(63.8)$ & \\
\hline Presence & $20(42.6)$ & $17(36.2)$ & \\
\hline \multicolumn{4}{|c|}{ Lymph node metastasis } \\
\hline N1 & $14(29.8)$ & $17(36.2)$ & \\
\hline $\mathrm{N} 2$ & $20(42.6)$ & $17(36.2)$ & \\
\hline N3 & $13(27.7)$ & $13(27.7)$ & \\
\hline \multicolumn{4}{|c|}{ Metastasis location } \\
\hline Absence & $0(0.0)$ & $4(8.5)$ & \\
\hline Liver & $29(61.7)$ & $28(59.6)$ & \\
\hline Lung & $11(23.4)$ & $10(21.3)$ & \\
\hline Multiple organ & 7 (14.9) & 5 (10.6) & \\
\hline
\end{tabular}

${ }^{\mathrm{a} A l l}$ patients and $\mathrm{HC}$ individuals were female. EOC, epithelial ovarian cancer; EOC/DM, EOC with metastasis; EOC/NDM, EOC without metastasis; HC, healthy control; TNM, tumor-node-metastasis; CA125, carbohydrate antigen 125.

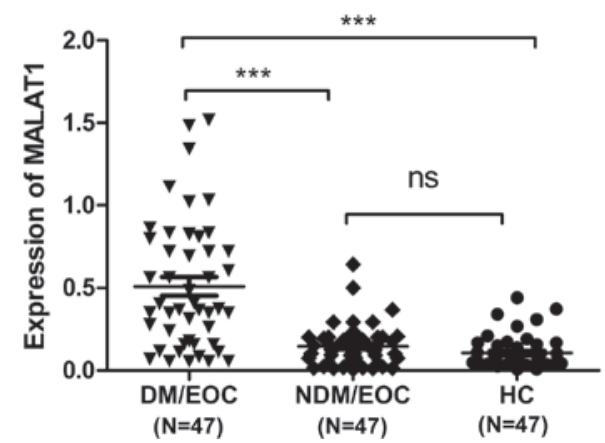

Figure 1. Plasma MALAT1 levels in EOC patients and HC individuals. The levels of plasma MALAT1 were determined by reverse transcription-quantitative polymerase chain reaction. Data are expressed as the mean $\log 102^{-\Delta \Delta C t}$ relative to the control $\beta$-actin. The horizontal lines indicate the median values. ${ }^{* * *} \mathrm{P}<0.0001$. MALAT1, metastasis-associated lung adenocarcinoma transcript 1; EOC, epithelial ovarian carcinoma; HC, healthy control; DM/EOC, EOC with metastasis; NDM/EOC, EOC without metastasis; ns, not significant.

in the EOC/DM group $(\mathrm{P}<0.001$ vs. EOC/NDM and $\mathrm{HC}$; Fig. 1). In addition, Youden's index (sensitivity plus specificity minus 1) was chosen as the optimal cutoff value and ROC curves were constructed to estimate the diagnostic value of MALAT1 in discriminating distant metastasis in EOCs. ROC analyses indicated that plasma MALAT1 yielded an AUC of 0.820 [95\% confidence interval (CI), 0.734-0.905; $\mathrm{P}<0.001$ ], with $87.2 \%$ sensitivity and $72.3 \%$ specificity for distinguishing EOC/DM from EOC/NDM. ROC analysis also yielded an AUC of 0.884 (95\% CI, 0.820-0.949; P<0.001), with $89.4 \%$ sensitivity and $72.3 \%$ specificity for distinguishing EOC/DM from HC. The plasma levels of MALAT1 were effectively distinguished in patients with EOC/DM from those with EOC/NDM or HC individuals (Fig. 2).

Cox analysis and Kaplan-Meier survival analysis of prognostic factors in EOC. Effect on the prognosis of plasma MALAT1 expression and other clinicopathological factors was also assessed. Low levels of CA125 were considered as $\leq 35 \mathrm{U} / \mathrm{ml}$, while high levels of CA125 were $>35 \mathrm{U} / \mathrm{ml}$. Low levels of plasma MALAT1 were considered as a fold-change of $<2$, while high levels of plasma MALAT1 were a fold-change of $>2$. Univariate and multivariate Cox analysis revealed that poor differentiation, TNM stage IV, N3 lymph node metastasis, presence of peritoneal 
Table II. Univariate and multivariate analysis of plasma MALAT1 and clinicopathological factors associated with survival in epithelial ovarian cancer patients.

\begin{tabular}{|c|c|c|c|c|c|c|}
\hline \multirow[b]{2}{*}{ Variable } & \multicolumn{3}{|c|}{ Univariate analysis } & \multicolumn{3}{|c|}{ Multivariate analysis } \\
\hline & Hazard ratio & $95 \% \mathrm{CI}$ & P-value & Hazard ratio & $95 \% \mathrm{CI}$ & P-value \\
\hline Differentiation (poor) & 3.576 & $1.356-6.754$ & 0.015 & 2.357 & $1.085-5.867$ & 0.023 \\
\hline TNM stage (IV) & 2.376 & $0.989-5.374$ & 0.005 & 1.864 & $0.956-4.356$ & 0.043 \\
\hline Metastasis location (lung) & 1.137 & $0.376-3.202$ & 0.082 & - & - & - \\
\hline Lymph node metastasis (N3) & 1.271 & $0.352-2.328$ & 0.043 & 1.479 & $0.561-4.546$ & 0.037 \\
\hline Peritoneal invasion (presence) & 2.725 & $0.934-6.347$ & 0.025 & 2.435 & $0.834-4.563$ & 0.039 \\
\hline Serum CA125 (>35 U/ml) & 2.259 & $0.563-3.194$ & 0.018 & 3.189 & $1.043-6.487$ & 0.017 \\
\hline Plasma MALAT1 (high) & 2.391 & $1.057-4.672$ & 0.031 & 3.322 & $1.140-5.680$ & 0.028 \\
\hline
\end{tabular}

TNM, tumor-node-metastasis; CA125, carbohydrate antigen 125; MALAT1, metastasis-associated lung adenocarcinoma transcript 1; CI, confidence interval.
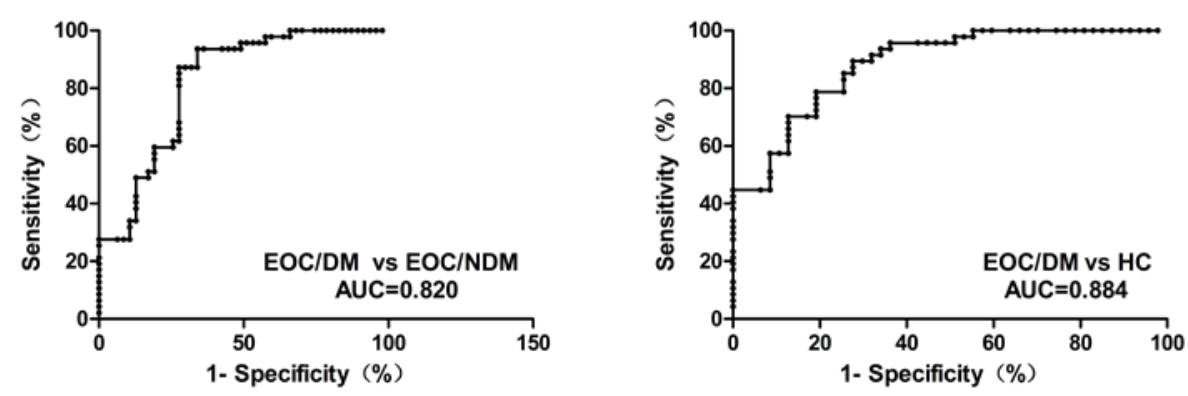

Figure 2. ROC analysis of the sensitivity and specificity of the levels of plasma MALAT1 in distinguishing DM/EOC from NDM/EOC and HC patients. The levels of plasma MALAT1 in the DM/EOC ( $n=47)$, NDM/EOC $(n=47)$ and HC $(n=47)$ groups were subjected to ROC analyses. The ROC-AUC for the levels of plasma MALAT1 to distinguish EOC/DM patients from EOC/NDM patients or HC individuals was 0.820 and 0.884 , respectively (P<0.001). MALAT1, metastasis-associated lung adenocarcinoma transcript 1; EOC, epithelial ovarian cancer; EOC/DM, EOC with metastasis; EOC/NDM, EOC without metastasis; $\mathrm{HC}$, healthy controls; AUC, area under the curve; ROC, receiver operating characteristic.

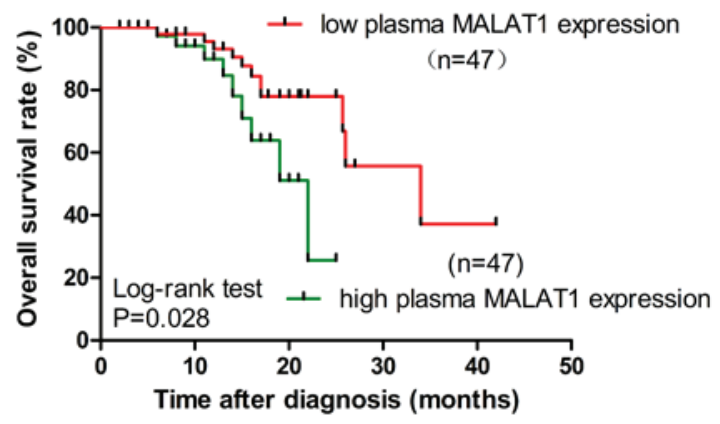

Figure 3. Kaplan-Meier survival curves for patients with EOC plotted for plasma MALAT1 levels. The P-value was calculated using the log-rank test between patients with high fold-change $(>2)$ and low fold-change $(<2)$. The OS rate of patients with EOC with low plasma MALAT1 expression was significantly higher than the OS in the patients with high plasma MALAT expression $(\mathrm{P}=0.028)$. EOC, epithelial ovarian cancer; OS, overall survival; MALAT1, metastasis-associated lung adenocarcinoma transcript 1.

invasion, high serum CA125 levels and high plasma MALAT1 levels were significantly associated with short life expectancy (Table II). Using the Kaplan-Meier and log-rank methods, it was found that high expression of the plasma MALAT1 was associated with a shorter overall survival (OS) of patients with EOC (hazard ratio, 3.322; 95\% CI, 1.140-5.680; $\mathrm{P}=0.028$; Fig. 3). Upregulation of plasma MALAT1 may independently contribute to a poor prognosis in patients with EOC.

\section{Discussion}

EOC ranks among the most common and lethal malignant diseases in gynecological cancers. Due to the location of the ovaries and the biological characteristics of insidious onset of EOC, it becomes extremely challenging to diagnose ovarian cancer at an early curable stage. In total, $>70 \%$ of patients present with advanced disease at diagnosis (34). The cure rate of women with advanced ovarian cancer is $\leq 40 \%$ as a result of challenging and inefficient surgery on metastatic disease (35). Poor prognosis of EOC is associated with the early onset of tumor metastasis. The mechanisms of EOC metastasis development are poorly understood and require additional exploration. Several studies have found that abnormal expression of lncRNAs are involved in tumor metastasis through regulating the expression of other molecules (36-39). Ji et al (22) identified the MALAT1 lncRNA by subtractive hybridization, establishing it as a prognostic marker for metastasis and survival in NSCLC. However, the roles of MALAT1 in the metastasis of EOC have yet to be fully elucidated. 
Due to the features of IncRNAs, such as long fragments (>200 nt), easy degradation in plasma, and extremely low concentration of total RNA in plasma, the current detection of plasma lncRNA as tumor markers becomes extremely challenging. Arita et al found that the lncRNA full-length form is not stable in plasma (40), but certain fragments in the plasma are highly stable and abundant $(32,41,42)$. The present study assessed the relevance of plasma MALAT1 to metastasis of EOC. The results demonstrated that a specific stable MALAT1 fragment existed in plasma, which was identified by a previous study (43), thus making circulating lncRNA expression detection available.

The present results showed that the level of plasma MALAT1 was markedly increased in patients with EOC/DM compared with patients with EOC/NDM and the HC group, and could effectively identify patients in the EOC/DM group from those in the EOC/NDM and $\mathrm{HC}$ group, thus indicating a suitable plasma biomarker for MALAT1 in EOC metastasis development. Furthermore, through Kaplan-Meier survival analysis and Cox analysis, it was found that elevated MALAT1 expression was associated with a shorter OS time in patients with EOC, as well as poor differentiation, advanced-stage disease, multiple lymph node metastases, presence of peritoneal invasion and increased serum CA125 level. Although the mechanism remains to be elucidated, these findings indicated that MALAT1 may play an important role in the cancer metastatic process, and may be a useful novel marker for metastatic EOC. Similar to the present findings, Shen et al (44) found that the level of MALAT1 was significantly increased in brain metastasis compared with non-brain metastasis samples, and subsequent functional studies indicated that MALAT1 promoted lung cancer cell brain metastasis by inducing epithelial-mesenchymal transition. Several studies have revealed that there are other MALAT1-mediated molecular pathways involved in tumor metastasis, such as through binding to splicing factor, proline- and glutamine-rich (SFPQ) and releasing the oncogene polypyrimidine tract binding protein 2 (PTBP2) from the SFPQ/PTBP2 complex (25), activating the extracellular signal-regulated kinase/Mitogenactivated protein kinase or phosphoinositide 3-kinase/AKT pathway to result in distant metastasis in numerous cancer types $(26,28)$. Overall, these mechanisms may explain why the present patients with high expression of MALAT1 expression demonstrated a strong metastasis tendency.

In summary, the high heterogeneity of metastatic EOC leads to resistance to chemotherapy drugs, poor prognosis and lack of effective targeting therapy. Therefore, identifying specific EOC metastasis-associated markers, and subsequently executing an early intervention have become the 'bottleneck' problem of EOC treatment. In the present study, it was found that plasma MALAT1 upregulation was closely associated with EOC distant metastasis and may be an independent risk factor for poor prognosis. The results of the present study may aid in understanding the molecular mechanisms of EOC with metastasis, which possess potential clinical value for metastatic EOC screening and early diagnosis, and may be a potential target for the treatment of EOC with metastasis.

\section{References}

1. Jemal A, Bray F, Center MM, Ferlay J, Ward E and Forman D: Global cancer statistics. CA Cancer J Clin 61: 69-90, 2011.
2. Parkin DM, Bray F, Ferlay J and Pisani P: Global cancer statistics, 2002. CA Cancer J Clin 55: 74-108, 2005.

3. Chan JK, Cheung MK, Husain A, Teng NN, West D, Whittemore AS, Berek JS and Osann K: Patterns and progress in ovarian cancer over 14 years. Obstet Gynecol 108: 521-528, 2006.

4. Prat J: New insights into ovarian cancer pathology. Ann Oncol 23 (Suppl 10): x111-x117, 2012.

5. Jelovac D and Armstrong DK: Recent progress in the diagnosis and treatment of ovarian cancer. CA Cancer J Clin 61: 183-203, 2011.

6. Herzog TJ and Pothuri B: Ovarian cancer: A focus on management of recurrent disease. Nat Clin Pract Oncol 3: 604-611, 2006.

7. Siegel R, Ma J, Zou Z and Jemal A: Cancer statistics, 2014. CA Cancer J Clin 64: 9-29, 2014.

8. Greenlee RT, Hill-Harmon MB, Murray T and Thun M: Cancer statistics, 2001. CA Cancer J Clin 51: 15-36, 2001.

9. Hoskins P, Eisenhauer E, Vergote I, Dubuc-Lissoir J, Fisher B, Grimshaw R, Oza A, Plante M, Stuart G and Vermorken J: Phase II feasibility study of sequential couplets of Cisplatin/Topotecan followed by paclitaxel/cisplatin as primary treatment for advanced epithelial ovarian cancer: A national cancer institute of canada clinical trials group study. J Clin Oncol 18: 4038-4044, 2000.

10. Theriault C, Pinard M, Comamala M, Migneault M, Beaudin J, Matte I, Boivin M, Piché A and Rancourt C: MUC16 (CA125) regulates epithelial ovarian cancer cell growth, tumorigenesis and metastasis. Gynecol Oncol 121: 434-443, 2011.

11. Dorigo O and Berek JS: Personalizing CA125 levels for ovarian cancer screening. Cancer Prev Res (Phila) 4: 1356-1359, 2011.

12. Nakajo M, Nakajo M, Nakayama H, Jinguji M, Nakabeppu Y, Higashi M, Nakamura Y, Sato M and Yoshiura T: Dexamethasone suppression FDG PET/CT for differentiating between true- and false-positive pulmonary and mediastinal lymph node metastases in non-small cell lung cancer: A pilot study of FDG PET/CT after oral administration of dexamethasone. Radiology 279: 246-253, 2016.

13. Fujimoto A, Furuta M, Totoki Y, Tsunoda T, Kato M, Shiraishi Y, Tanaka H, Taniguchi H, Kawakami Y, Ueno M, et al: Whole-genome mutational landscape and characterization of noncoding and structural mutations in liver cancer. Nat Genet 48: 500-509, 2016.

14. Chen D, Sun Q, Cheng X, Zhang L, Song W, Zhou D, Lin J and Wang W: Genome-wide analysis of long noncoding RNA (lncRNA) expression in colorectal cancer tissues from patients with liver metastasis. Cancer Med: May 11, 2016 (Epub ahead of print).

15. Huarte M: The emerging role of lncRNAs in cancer. Nat Med 21: 1253-1261, 2015

16. Prensner JR and Chinnaiyan AM: The emergence of lncRNAs in cancer biology. Cancer Discov 1: 391-407, 2011.

17. Cao B, Song N, Zhang M, Di C, Yang Y, Lu Y, Chen R, Lu ZJ and Guo M: Systematic study of novel lncRNAs in different gastrointestinal cancer cells. Discov Med 21: 159-171, 2016.

18. Wang L, Li J,Zhao H, Hu J, Ping Y,Li F, Lan Y, Xu C, Xiao Y and Li $\mathrm{X}$ : Identifying the crosstalk of dysfunctional pathways mediated by lncRNAs in breast cancer subtypes. Mol Biosyst 12: 711-720, 2016.

19. Zheng HT, Shi DB, Wang YW, Li XX, Xu Y, Tripathi P, Gu WL, Cai GX and Cai SJ: High expression of lncRNA MALAT1 suggests a biomarker of poor prognosis in colorectal cancer. Int J Clin Exp Pathol 7: 3174-3181, 2014.

20. Pang EJ, Yang R, Fu XB and Liu YF: Overexpression of long non-coding RNA MALAT1 is correlated with clinical progression and unfavorable prognosis in pancreatic cancer. Tumour Biol 36: 2403-2407, 2015.

21. Zhang HM, Yang FQ, Chen SJ, Che J and Zheng JH: Upregulation of long non-coding RNA MALAT1 correlates with tumor progression and poor prognosis in clear cell renal cell carcinoma. Tumour Biol 36: 2947-2955, 2015.

22. Ji P, Diederichs S, Wang W, Böing S, Metzger R, Schneider PM, Tidow N, Brandt B, Buerger H, Bulk E, et al: MALAT-1, a novel noncoding RNA and thymosin beta4 predict metastasis and survival in early-stage non-small cell lung cancer. Oncogene 22: 8031-8041, 2003.

23. Shen L, Chen L, Wang Y, Jiang X, Xia H and Zhuang Z: Long noncoding RNA MALAT1 promotes brain metastasis by inducing epithelial-mesenchymal transition in lung cancer. J Neurooncol 121: 101-108, 2015.

24. Gutschner T, Hämmerle M, Eissmann M, Hsu J, Kim Y, Hung G, Revenko A, Arun G, Stentrup M, Gross M, et al: The noncoding RNA MALAT1 is a critical regulator of the metastasis phenotype of lung cancer cells. Cancer Res 73: 1180-1189, 2013. 
25. Ji Q, Zhang L, Liu X, Zhou L, Wang W, Han Z, Sui H, Tang Y, Wang Y, Liu N, et al: Long non-coding RNA MALAT1 promotes tumour growth and metastasis in colorectal cancer through binding to SFPQ and releasing oncogene PTBP2 from SFPQ/PTBP2 complex. Br J Cancer 111: 736-748, 2014.

26. Dong Y, Liang G, Yuan B, Yang C, Gao R and Zhou X: MALAT1 promotes the proliferation and metastasis of osteosarcoma cells by activating the PI3K/Akt pathway. Tumour Biol 36: 1477-1486, 2015.

27. Fan Y, Shen B, Tan M, Mu X, Qin Y, Zhang F and Liu Y: TGF- $\beta$-induced upregulation of malat 1 promotes bladder cancer metastasis by associating with suz12. Clin Cancer Res 20: $1531-1541,2014$.

28. Wu XS, Wang XA, Wu WG, Hu YP, Li ML, Ding Q, Weng H, Shu YJ, Liu TY, Jiang L, et al: MALAT1 promotes the proliferation and metastasis of gallbladder cancer cells by activating the ERK/MAPK pathway. Cancer Biol Ther 15: 806-814, 2014.

29. National Comprehensive Cancer Network. NCCN Clinical Practice Guidelines in Oncology: (NCCN Guidelines ${ }^{\circledR}$ ) Ovarian Cancer Including Fallopian Tube Cancer and Primary Peritoneal Cancer. Version 1.2015. http://www.nccn.org/professionals/ physician_gls/pdf/ovarian.pdf. Accessed May 5, 2016.

30. Edge SB, Byrd DR, Compton CC, Fritz AG, Greene FL and Trotti A (eds): Ovary and primary peritoneal carcinoma. In: AJCC Cancer Staging Manual. 7th edition. Springer-Verlag, NY, pp419-428, 2010.

31. Chen Q, Ge X, Zhang Y, Xia H, Yuan D, Tang Q, Chen L, Pang X, Leng W and Bi F: Plasma miR-122 and miR-192 as potential novel biomarkers for the early detection of distant metastasis of gastric cancer. Oncol Rep 31: 1863-1870, 2014.

32. Ren S, Wang F, Shen J, Sun Y, Xu W, Lu J, Wei M, Xu C, Wu C, Zhang Z, et al: Long non-coding RNA metastasis associated in lung adenocarcinoma transcript 1 derived miniRNA as a novel plasma-based biomarker for diagnosing prostate cancer. Eur J Cancer 49: 2949-2959, 2013.

33. Livak KJ and Schmittgen TD: Analysis of relative gene expression data using real-time quantitative PCR and the $2^{-\Delta \Delta C T}$ method. Methods 25: 402-408, 2001.

34. Barakat RR, Markman M and Randall M (eds): Principles and Practice of Gynecologic Oncology. 5th edition. Lippincott Williams \& Wilkins, 2009.

35. Siegel R, Naishadham D and Jemal A: Cancer statistics, 2013. CA Cancer J Clin 63: 11-30, 2013.
36. Huang JF, Guo YJ, Zhao CX, Yuan SX, Wang Y, Tang GN, Zhou WP and Sun SH: Hepatitis B virus X protein (HBx)-related long noncoding RNA (lncRNA) down-regulated expression by HBx (Dreh) inhibits hepatocellular carcinoma metastasis by targeting the intermediate filament protein vimentin. Hepatology 57: 1882-1892, 2013.

37. Li H, Yu B, Li J, Su L, Yan M, Zhu Z and Liu B: Overexpression of 1ncRNA H19 enhances carcinogenesis and metastasis of gastric cancer. Oncotarget 5: 2318-2329, 2014.

38. Wang Y, Liu X, Zhang H, Sun L, Zhou Y, Jin H, Zhang H, Zhang H, Liu J, Guo H, et al: Hypoxia-inducible lncRNA-AK058003 promotes gastric cancer metastasis by targeting $\gamma$-synuclein. Neoplasia 16: 1094-1106, 2014.

39. Zhu M, Chen Q, Liu X, Sun Q, Zhao X, Deng R, Wang Y, Huang J, Xu M, Yan J and Yu J: lncRNA H19/miR-675 axis represses prostate cancer metastasis by targeting TGFBI. FEBS J 281: 3766-3775, 2014.

40. Arita T, Ichikawa D, Konishi H, Komatsu S, Shiozaki A, Shoda K, Kawaguchi T, Hirajima S, Nagata H, Kubota T, et al: Circulating long non-coding RNAs in plasma of patients with gastric cancer. Anticancer Res 33: 3185-3193, 2013.

41. Tsui NB, Ng EK and Lo YM: Stability of endogenous and added RNA in blood specimens, serum and plasma. Clin Chem 48: 1647-1653, 2002.

42. Ng EK, Tsui NB, Lau TK, Leung TN, Chiu RW, Panesar NS, Lit LC, Chan KW and Lo YM: mRNA of placental origin is readily detectable in maternal plasma. Proc Natl Acad Sci USA 100: 4748-4753, 2003.

43. Weber DG, Johnen G, Casjens S, Bryk O, Pesch B, Jöckel KH, Kollmeier $\mathrm{J}$ and Brüning T: Evaluation of long noncoding RNA MALAT1 as a candidate blood-based biomarker for the diagnosis of non-small cell lung cancer. BMC Res Notes 6: 518, 2013.

44. Shen L, Chen L, Wang Y, Jiang X, Xia H and Zhuang Z: Long noncoding RNA MALAT1 promotes brain metastasis by inducing epithelial-mesenchymal transition in lung cancer. J Neurooncol 121: 101-108, 2015. 